\title{
Path Integral for Confined Dirac Fermions in a Constant Magnetic Field
}

\author{
Abdeldjalil Merdaci $^{* a, b}$, Ahmed Jellal ${ }^{\dagger c, d}$ and Lyazid Chetouani ${ }^{b}$ \\ ${ }^{a}$ Physics Department, College of Science, King Faisal University, \\ PO Box 380, Alahsa 31982, Saudi Arabia \\ ${ }^{b}$ Département de Physique, Faculté des Sciences Exactes, Université Mentouri, \\ 25000 Constantine, Algérie. \\ ${ }^{c}$ Saudi Center for Theoretical Physics, Dhahran, Saudi Arabia \\ ${ }^{d}$ Theoretical Physics Group, Faculty of Sciences, Chouaïb Doukkali University, \\ PO Box 20, 24000 El Jadida, Morocco
}

\begin{abstract}
We consider Dirac fermion confined in harmonic potential and submitted to a constant magnetic field. The corresponding solutions of the energy spectrum are obtained by using the path integral techniques. For this, we begin by establishing a symmetric global projection, which provides a symmetric form for the Green function. Based on this, we show that it is possible to end up with the propagator of the harmonic oscillator for one charged particle. After some transformations, we derive the normalized wave functions and the eigenvalues in terms of different physical parameters and quantum numbers. By interchanging quantum numbers, we show that our solutions have interesting properties. The density of current and the non-relativistic limit are analyzed where different conclusions are obtained. Finally, the completeness of the Dirac oscillator eigenfunctions is proved by using the standard properties of the generalized Laguerre polynomials.
\end{abstract}

PACS numbers: 03.65.Pm, 03.65.Ge

Keywords: Dirac equation, confinement, magnetic field, path integral.

*amerdaci@kfu.edu.sa

†ajellal@ictp.it - a.jellal@ucd.ac.ma 


\section{Introduction}

The Dirac equation in (2+1)-dimensions is an important tool not only from mathematical point of view but also from the large applications in physics. In particular, several condensed matter phenomena point out to the existence of a $(2+1)$-dimensional energy spectrum determined by the relativistic Dirac equation [1]. For very recent works, one may consult references [2-7] and for early works relevant to our subject we cite [8,9]. As example graphene [10], which is a single layer of carbon atoms arranged into a planar honeycomb lattice. This system has attracted a considerable attention from both experimental and theoretical researchers since its experimental realization in 2004 [11]. This is because of its unique and outstanding mechanical, electronic, optical, thermal and chemical properties [12]. Most of these marvelous properties are due to the apparently relativistic-like nature of its carriers, electrons behave as massless Dirac fermions in graphene systems. In fact starting from the original tight-binding Hamiltonian describing graphene it has been shown theoretically that the low-energy excitations of graphene appear to be massless chiral Dirac fermions.

On the other hand, due to recent technological advances in nano-fabrication there were a lot of interest in the study of low dimensional quantum systems such as quantum wells, quantum wires and quantum dots [13]. In particular, there has been considerable amount of work in recent years on semiconductors confined structures, which finds applications in electronic and optoelectronic devices. These show the relevance of the confinements in physics and therefore deserve to be analyzed in other systems like relativistic ones. Furthermore, an applied magnetic field perpendicular to the heterostructure systems quantizes the energy levels in the plane, drastically affecting the density of states giving rise to the famous quantum Hall effect [14].

A confined relativistic system subjected to a constant magnetic field was studied normal to the plane in [15]. In fact, the exact solution of the Dirac equation in $(2+1)$-dimensions was obtained depending on various parameters. The solution space consists of positive and negative energy solutions, each of which splits into two disconnected subspaces depending on the sign of an azimuthal quantum number $l=0, \pm 1, \pm 2, \ldots$ and whether the cyclotron frequency is larger or smaller than the oscillator frequency. The spinor wave function was written in terms of the associated Laguerre polynomials. For negative $l$, the relativistic energy spectrum was found to be in finitely degenerate due to the fact that it is dependent of $l$.

Motivated by different investigations on the Dirac fermions in $(2+1)$-dimensions, we give an exact solution of a problem that has been studied at various levels by researchers dealing with different physical phenomena. More precisely, we return to the problem studied in [15] and use another techniques to determine the solutions of the energy spectrum as well dealing with related issues. Then, we consider a relativistic particle subjected to an external magnetic field as well as to a confining potential. By using the path integral formalism and making different transformations, we show that the causal Green function can be written in terms of the propagator for the harmonic oscillator for a charged particle. This allowed us to obtain various solutions, in terms of different physical parameters and quantum numbers, and emphasis similarities to, and differences from, already published work elsewhere $[15,16]$.

Furthermore, we give discussions of our results based on different physical settings. The full rich space of solutions suggested enabled us to carry out a deeper analysis in relation to various physical 
quantities. For instance, we show that our energy remains invariant under the changes of the quantum numbers characterizing our system behavior, reveals a non trivial symmetry of the problem. Also, we obtain, as expected in the absence of applied voltage, a null current density for both directions in the Cartesian representation. However, this is not the case in polar coordinate. In fact, we show that the radial current vanishes whereas the angular component does not. It is dependent on various physical parameters in the problem. Additionally, we discuss the non-relativistic limit of the problem.

The paper is organized as follows. In section 2, we show that how one can use the global projection rather than local one for $(2+1)$-dimensional Dirac equation in electromagnetic field. In section 3 , we give the theoretical formulation of the problem where different changes are introduced to simplify the process for obtaining the solutions of the energy spectrum. More precisely, we will use the causal Green function as well as different techniques to solve our problem. We determine the eigenvalues and eigenspinors in terms of different physical parameters and quantum numbers in section 4 . In section 5 , we analysis our results by showing that our system has hidden symmetries, those can be used to deal with different issues. In section 6 , we calculate the density current in polar coordinate system. We show how to recover the nonrelativistic limit from our results in section 7 . The proof of the completeness relation of the Dirac oscillator eigenfunctions will be presented in section 8 . We conclude our findings in the final section.

\section{Symmetric global projection}

Before embarking on our problem and investigate its basic features, let us establish some mathematical tool that needed to deal with different issues. For this, we start by considering a relativistic charged particle in electromagnetic field to show that the global projection giving rises to an new symmetric form for the Green function. For such system the causal Green function $S^{c}\left(\mathbf{x}_{b}, \mathbf{x}_{a}\right)$ satisfies the two Dirac equations [17]

$$
\begin{aligned}
& \left(\gamma^{\mu}\left(i \partial_{b \mu}-e A_{\mu}\left(\mathbf{x}_{b}\right)\right)-m\right) S^{c}\left(\mathbf{x}_{b}, \mathbf{x}_{a}\right)=\delta^{3}\left(\mathbf{x}_{b}-\mathbf{x}_{a}\right) \\
& S^{c}\left(\mathbf{x}_{b}, \mathbf{x}_{a}\right)\left(\gamma^{\mu}\left(-i \overleftarrow{\partial}_{a \mu}-e A_{\mu}\left(\mathbf{x}_{a}\right)\right)-m\right)=\delta^{3}\left(\mathbf{x}_{b}-\mathbf{x}_{a}\right)
\end{aligned}
$$

where the matrices $\gamma^{\mu}$ are defined by the relations generating Clifford algebra

$$
\left[\gamma^{\mu}, \gamma^{\nu}\right]=-2 i \sigma^{\mu \nu}, \quad\left\{\gamma^{\mu}, \gamma^{\nu}\right\}=2 \eta^{\mu \nu}
$$

Actually, we have $\eta^{\mu \nu}=\operatorname{diag}(1,-1,-1)$, with $(\mu, \nu=0,1,2)$ and $\gamma^{\mu}=\frac{i}{2} \epsilon^{\mu \nu \lambda} \gamma_{\nu} \gamma_{\lambda}$ or equivalently

$$
\gamma^{0}=i \gamma^{1} \gamma^{2}, \quad \gamma^{1}=-i \gamma^{0} \gamma^{2}, \quad \gamma^{2}=i \gamma^{0} \gamma^{1}
$$

Formally, $S^{c}\left(\mathbf{x}_{b}, \mathbf{x}_{a}\right)$ is the matrix element in the coordinate space

$$
S^{c}\left(\mathbf{x}_{b}, \mathbf{x}_{a}\right)=\left\langle\mathbf{x}_{b}\left|S^{c}\right| \mathbf{x}_{a}\right\rangle
$$

of the inverse Dirac operator $O_{-}^{-1}$

$$
S^{c}=\left(\gamma^{\mu}\left(p_{\mu}-e A_{\mu}\right)-m\right)^{-1}=O_{-}^{-1}=O_{+}\left(O_{-} O_{+}\right)^{-1}
$$


where the operators $O_{ \pm}$are given by

$$
O_{ \pm}=\gamma^{\mu}\left(p_{\mu}-e A_{\mu}\right) \pm m
$$

We recall that Alexandrou et al. [18] have been described a massive Dirac particle in external vector and scalar fields by making use of the asymmetric form. In the present study, we consider the symmetric form to write the Dirac propagator in order to straightforwardly derive the corresponding spinors. Indeed, the local inverse Dirac operator (6) can be written in two equivalent forms

$$
S^{c}=\left(\gamma^{\mu}\left(p_{\mu}-e A_{\mu}\right)-m\right)^{-1}=O_{-}^{-1}=O_{+} S_{g}^{c}=S_{g}^{c} O_{+}
$$

where the Green operator for global projection is

$$
S_{g}^{c}=\left(O_{-} O_{+}\right)^{-1}=\left(O_{+} O_{-}\right)^{-1}
$$

and the label $g$ stands for global. It is clear that the matrix element of $S_{g}^{c}$ verifies the quadratic Dirac equation

$$
O_{-} O_{+} S_{g}^{c}\left(\mathbf{x}_{b}, \mathbf{x}_{a}\right)=O_{+} O_{-} S_{g}^{c}\left(\mathbf{x}_{b}, \mathbf{x}_{a}\right)=\delta^{3}\left(\mathbf{x}_{b}-\mathbf{x}_{a}\right) .
$$

Now let us insert the completeness relation $\int|z\rangle\langle z| d^{3} z=\mathbb{I}$ in the matrix element (5) and use (8) to end up with

$$
S^{c}\left(\mathbf{x}_{b}, \mathbf{x}_{a}\right)=\int\left\langle x_{b}\left|O_{+}\right| z\right\rangle\left\langle z\left|S_{g}^{c}\right| x_{a}\right\rangle d^{3} z=\int\left\langle x_{b}\left|S_{g}^{c}\right| z\right\rangle\left\langle z\left|O_{+}\right| x_{a}\right\rangle d^{3} z
$$

which implies the equality

$$
\left(\gamma^{\mu} \vec{\pi}_{\mu}(b)+m\right) S_{g}^{c}\left(\mathbf{x}_{b}, \mathbf{x}_{a}\right)=S_{g}^{c}\left(\mathbf{x}_{b}, \mathbf{x}_{a}\right)\left(\gamma^{\mu} \overleftarrow{\pi}_{\mu}(a)+m\right)
$$

where the momentum operators read as

$$
\vec{\pi}_{\mu}=i \frac{\vec{\partial}}{\partial x^{\mu}}-e A_{\mu}(x), \quad \overleftarrow{\pi}_{\mu}=-i \frac{\overleftarrow{\partial}}{\partial x^{\mu}}-e A_{\mu}(x)
$$

The right and left derivatives are defined by

$$
\hat{A} \frac{\vec{\partial}}{\partial x} \hat{B}=\hat{A}\left(\frac{\partial}{\partial x} \hat{B}\right), \quad \hat{A} \frac{\overleftarrow{\partial}}{\partial x} \hat{B}=\left(\frac{\partial}{\partial x} \hat{A}\right) \hat{B}
$$

Therefore, we can deduce the following new symmetric form

$$
S^{c}\left(\mathbf{x}_{b}, \mathbf{x}_{a}\right)=\frac{1}{2}\left(\gamma^{\mu} \vec{\pi}_{\mu}(b)+m\right) S_{g}^{c}\left(\mathbf{x}_{b}, \mathbf{x}_{a}\right)+\frac{1}{2} S_{g}^{c}\left(\mathbf{x}_{b}, \mathbf{x}_{a}\right)\left(\gamma^{\mu} \overleftarrow{\pi}_{\mu}(a)+m\right)
$$

which is an interesting result and can be generalized to any dimension. We will see how it can be used to determine explicitly the solution of the energy spectrum.

\section{Application to confined system}

redTo be specific, let us consider a confined system of $(2+1)$-dimensional Dirac fermions in a constant magnetic field $\vec{B}$. This confinement can be realized by modifying the operators $O_{ \pm}$, given in (7), by 
the non-minimal substitution such that $\vec{p} \longrightarrow \vec{p}-i m \omega \vec{r} \gamma^{0}$, where $\omega$ is the oscillator frequency. By choosing the symmetric gauge the symmetric gauge $\vec{A}=\frac{B}{2}(-y, x)$, we can write (7)

$$
O_{ \pm}=\gamma^{0} p_{0}-\gamma^{1}\left(p_{x}-\frac{|e| B}{2} y-i m \omega x \gamma^{0}\right)-\gamma^{2}\left(p_{y}+\frac{|e| B}{2} x-i m \omega y \gamma^{0}\right) \pm m
$$

and using $\gamma^{0}=i \gamma^{1} \gamma^{2}$ to obtain in the compact form

$$
O_{ \pm}=\gamma^{0} p_{0}-\gamma^{1}\left(p_{x}+G y\right)-\gamma^{2}\left(p_{y}-G x\right) \pm m
$$

where we have set the parameter $G=m\left(\omega-\omega_{c}\right)$ and the cyclotron frequency $\omega_{c}=\frac{|e| B}{2 m} \neq \omega$. It is clearly seen that both frequencies $\omega$ and $\omega_{c}$ come from different sources but regrouped in he same place, which is an unique property of $(2+1)$-dimensional space.

Now, by using Schwinger proper time representation, we can express $S_{g}^{c}\left(\mathbf{x}_{b}, \mathbf{x}_{a}\right)$ in an integral expressions form

$$
S_{g}^{c}\left(\mathbf{x}_{b}, \mathbf{x}_{a}\right)=\left\langle\mathbf{x}_{b}\left|\left(O_{+} O_{-}\right)^{-1}\right| \mathbf{x}_{a}\right\rangle=-\frac{i}{2} \int_{0}^{\infty} d \lambda\left\langle\mathbf{x}_{b}\left|\exp \left(i \frac{\lambda}{2}(\mathcal{H}+i \varepsilon)\right)\right| \mathbf{x}_{a}\right\rangle
$$

where the Hamiltonian $\mathcal{H}$ is given by

$$
\mathcal{H}=O_{+} O_{-}=\hat{p}_{0}^{2}-\hat{p}_{x}^{2}-\hat{p}_{y}^{2}-G^{2}\left(\hat{x}^{2}+\hat{y}^{2}\right)-m^{2}+2 G\left(\hat{x} \hat{p}_{y}-\hat{y} \hat{p}_{x}\right)+2 i G \gamma^{1} \gamma^{2}
$$

for $G \neq 0$. Similar to [19], we represent $S_{g}^{c}\left(\mathbf{x}_{b}, \mathbf{x}_{a}\right)$ via the path integral

$$
\begin{aligned}
S_{g}^{c}\left(\mathbf{x}_{b}, \mathbf{x}_{a}\right) & =-\frac{i}{2} \exp \left(i \gamma \cdot \frac{\partial_{l}}{\partial \theta}\right) \int_{0}^{\infty} d \lambda \int D \mathbf{x} D \mathbf{p} \int \mathcal{D} \psi \\
& \times \exp \left\{i \int _ { 0 } ^ { 1 } \left[-\dot{\mathbf{x}} \cdot \mathbf{p}+\frac{\lambda}{2}\left(\mathbf{p}^{2}-m^{2}-G^{2} \mathbf{x}^{2}\right)\right.\right. \\
& \left.\left.+2 G \mathbf{x} \times \mathbf{p}-8 i G \psi^{1} \psi^{2}-i \psi_{\mu} \dot{\psi}^{\mu}\right] d \tau+\psi_{\mu}(1) \psi^{\mu}(0)\right\}\left.\right|_{\theta=0}
\end{aligned}
$$

where $\mathbf{x}=(t, x, y)$ and $(\psi, \theta)$ refer respectively to even and odd variables. They satisfy boundary conditions

$$
\mathbf{x}(0)=\mathbf{x}_{a}, \quad \mathbf{x}(1)=\mathbf{x}_{b}, \quad \psi^{\mu}(0)+\psi^{\mu}(1)=\theta^{\mu} .
$$

The path integration measure

$$
\mathcal{D} \psi=D \psi\left[\int_{\psi(1)+\psi(0)=0} D \psi \exp \left(\int_{0}^{1} \psi_{\mu} \dot{\psi}^{\mu} d \tau\right)\right]^{-1}
$$

has been considered above. Note that, by integrating over the path $t$, we can see that the momentums become constants, i.e. $p_{0}=$ const.

To go further, let us simplify our formalism by changing the momentum variables to define two others as

$$
p_{x}=\bar{p}_{x}-G y, \quad p_{y}=\bar{p}_{y}+G x
$$

which allow to write

$$
\begin{aligned}
S_{g}^{c}\left(\mathbf{x}_{b}, \mathbf{x}_{a}\right) & =-\frac{i}{2} \exp \left(i \gamma \cdot \frac{\partial_{l}}{\partial \theta}\right) \int_{0}^{\infty} d \lambda \int_{-\infty}^{+\infty} \frac{d p_{0}}{2 \pi} e^{-i p_{0}\left(t_{b}-t_{a}\right)+\frac{i \lambda}{2}\left(p_{0}^{2}-m^{2}\right)} \int D x D y D \bar{p}_{x} D \bar{p}_{y} \\
& \times \int \mathcal{D} \psi \exp \left\{i \int _ { 0 } ^ { 1 } \left[\bar{p}_{x} \dot{x}+\bar{p}_{y} \dot{y}-\frac{\lambda}{2} \bar{p}_{x}^{2}-\frac{\lambda}{2} \bar{p}_{y}^{2}\right.\right. \\
& \left.\left.+G(x \dot{y}-y \dot{x})-4 i \lambda G \psi^{1} \psi^{2}-i \psi_{\mu} \dot{\psi}^{\mu}\right] d \tau+\psi_{\mu}(1) \psi^{\mu}(0)\right\}\left.\right|_{\theta=0}
\end{aligned}
$$


Now integrating over $\bar{p}_{x}$ and $\bar{p}_{y}$ to find

$$
\begin{aligned}
S_{g}\left(\mathbf{x}_{b}, \mathbf{x}_{a}\right) & =-\frac{i}{2} \exp \left(i \gamma \cdot \frac{\partial_{l}}{\partial \theta}\right) \int_{0}^{\infty} d \lambda \int_{-\infty}^{+\infty} \frac{d p_{0}}{2 \pi} e^{-i p_{0}\left(t_{b}-t_{a}\right)+\frac{i \lambda}{2}\left(p_{0}^{2}-m^{2}\right)} \int D x D y \\
& \times\left.\int \mathcal{D} \psi e^{\left\{i \int_{0}^{1}\left(\frac{\dot{x}^{2}}{2 \lambda}+\frac{\dot{y}^{2}}{2 \lambda}+G(x \dot{y}-y \dot{x})-4 i \lambda G \psi^{1} \psi^{2}-i \psi_{\mu} \dot{\psi}^{\mu}\right) d \tau+\psi_{\mu}(1) \psi^{\mu}(0)\right\}}\right|_{\theta=0} .
\end{aligned}
$$

In order to integrate over $\psi^{\mu}(\tau)$, let us first eliminate the constants $\theta^{\mu}$ appearing in the boundary conditions (21) by performing the following changes

$$
\psi^{\mu}(\tau)=\xi^{\mu}(\tau)+\frac{1}{2} \theta^{\mu}, \quad \mu=0,1,2 .
$$

By replacing in (25), we obtain

$$
\begin{aligned}
S_{g}^{c}\left(\mathbf{x}_{b}, \mathbf{x}_{a}\right) & =-\frac{i}{2} \exp \left(i \gamma \cdot \frac{\partial_{l}}{\partial \theta}\right) \int_{0}^{\infty} d \lambda \int_{-\infty}^{+\infty} \frac{d p_{0}}{2 \pi} e^{-i p_{0}\left(t_{b}-t_{a}\right)+\frac{i \lambda}{2}\left(p_{0}^{2}-m^{2}\right)} \int D x D y \\
& \times \int \mathcal{D} \xi \exp \left\{i \int_{0}^{1}\left[\frac{\dot{x}^{2}}{2 \lambda}+\frac{\dot{y}^{2}}{2 \lambda}+G(x \dot{y}-y \dot{x})\right\}\right. \\
& \left.\left.-4 i \lambda G\left(\xi^{1} \xi^{2}-\frac{1}{2} \theta^{2} \xi^{1}(\tau)+\frac{1}{2} \theta^{1} \xi^{2}(\tau)+\frac{1}{4} \theta^{1} \theta^{2}\right)-i \xi_{\mu} \dot{\xi}^{\mu}\right] d \tau\right\}\left.\right|_{\theta=0}
\end{aligned}
$$

where the antiperiodic boundary conditions become

$$
\xi^{\mu}(1)+\xi^{\mu}(0)=0 .
$$

By considering $\xi=\left(\begin{array}{c}\xi^{1} \\ \xi^{2}\end{array}\right)$ and $\sigma_{2}=\left(\begin{array}{cc}0 & -i \\ i & 0\end{array}\right)$, one can easily see the relation

$$
\xi^{1} \xi^{2}=\frac{i}{2} \xi \sigma_{2} \xi
$$

Now using (29) and being able to undertake easily the integration over Grassmann variables, one can separate $(27)$ as

$$
\begin{aligned}
S_{g}^{c}\left(\mathbf{x}_{b}, \mathbf{x}_{a}\right) & =-\frac{i}{2} \exp \left(i \gamma \cdot \frac{\partial_{l}}{\partial \theta}\right) \int_{0}^{\infty} d \lambda \int_{-\infty}^{+\infty} \frac{d p_{0}}{2 \pi} e^{-i p_{0}\left(t_{b}-t_{a}\right)+\frac{i \lambda}{2}\left(p_{0}^{2}-m^{2}\right)} \\
& \times\left.\iint D x D y \exp \left\{i \int_{0}^{1}\left(\frac{\dot{x}^{2}}{2 \lambda}+\frac{\dot{y}^{2}}{2 \lambda}+G(x \dot{y}-y \dot{x})\right)\right\} \mathcal{I}(\lambda, \theta)\right|_{\theta=0}
\end{aligned}
$$

where the fermionic part is given by

$$
\begin{aligned}
\mathcal{I}(\lambda, \theta) & =e^{\lambda G \theta^{1} \theta^{2}} \int \mathcal{D} \xi^{0} \iint \mathcal{D}^{2} \xi \\
& \times e^{\int_{0}^{1} \xi(\tau) \mathcal{R}\left(\lambda \mid \tau, \tau^{\prime}\right) \xi\left(\tau^{\prime}\right) d \tau d \tau^{\prime}+\int_{0}^{1} \mathcal{J}(\tau) \xi(\tau) d \tau+\int_{0}^{1} \xi^{0}(\tau) \delta^{\prime}\left(\tau-\tau^{\prime}\right) \xi^{0}\left(\tau^{\prime}\right) d \tau d \tau^{\prime}}
\end{aligned}
$$

and we have

$$
\mathcal{R}\left(\lambda \mid \tau, \tau^{\prime}\right)=-\delta^{\prime}\left(\tau-\tau^{\prime}\right)+2 i \lambda G \sigma_{2} \delta\left(\tau-\tau^{\prime}\right), \quad \mathcal{J}^{T}(\tau)=2 \lambda G\left(-\theta^{2}, \theta^{1}\right) .
$$

Since the integration over $\xi^{0}$ yields trivially the unity, then integrating over $\xi^{1}$ and $\xi^{2}$ to obtain

$$
\mathcal{I}(x, y, \lambda ; \theta)=\sqrt{\frac{\operatorname{det} \mathcal{R}(\lambda)}{\operatorname{det} \mathcal{R}(0)}} e^{\lambda G \theta^{1} \theta^{2}} e^{\frac{1}{4} \int_{0}^{1} \int_{0}^{1} \mathcal{J}(\tau) \cdot \mathcal{R}^{-1}\left(\lambda \mid \tau, \tau^{\prime}\right) \cdot \mathcal{J}\left(\tau^{\prime}\right) d \tau d \tau^{\prime}}
$$


where $\mathcal{R}^{-1}\left(\lambda \mid \tau, \tau^{\prime}\right)$ is the inverse matrix of $\mathcal{R}\left(\lambda \mid \tau, \tau^{\prime}\right)$, which can be considered as an operator acting on the space of antiperiodic functions

$$
\mathcal{R}^{-1}(\lambda \mid 1, \tau)+\mathcal{R}^{-1}(\lambda \mid 0, \tau)=0, \quad \forall \tau \in[0,1]
$$

It obeys the first order differential equation

$$
\left(-\frac{\partial}{\partial \tau}+2 i G \lambda \sigma_{2}\right) \mathcal{R}^{-1}\left(\lambda \mid \tau, \tau^{\prime}\right)=\delta\left(\tau-\tau^{\prime}\right)
$$

giving the solution

$$
\mathcal{R}^{-1}\left(\lambda \mid \tau, \tau^{\prime}\right)=\frac{1}{2} e^{2 i \lambda G \sigma_{2}\left(\tau-\tau^{\prime}\right)}\left[i \sigma_{2} \tan (\lambda G)-\varepsilon\left(\tau-\tau^{\prime}\right)\right], \quad \varepsilon\left(\tau-\tau^{\prime}\right)=\operatorname{sgn}\left(\tau-\tau^{\prime}\right) .
$$

Using similar calculations as in [20] to get

$$
\begin{aligned}
\sqrt{\frac{\operatorname{det} \mathcal{R}(\lambda)}{\operatorname{det} \mathcal{R}(0)}} & =e^{\frac{1}{2} \operatorname{Tr} \int_{0}^{\lambda} \mathcal{R}^{-1}\left(\lambda^{\prime}\right) \frac{d}{d \lambda^{\prime}} \mathcal{R}\left(\lambda^{\prime}\right) d \lambda^{\prime}}=e^{\frac{1}{2} \operatorname{Tr} \int_{0}^{\lambda}\left[\frac{1}{2} i \sigma_{2} \tan \left(\lambda^{\prime} G\right)\right]\left[2 i G \sigma_{2}\right] d \lambda^{\prime}} \\
& =e^{-\int_{0}^{\lambda} \tan \left(\lambda^{\prime} G\right) G d \lambda^{\prime}}=\cos (\lambda G) .
\end{aligned}
$$

Combining all to show that (30) takes the form

$$
S_{g}^{c}\left(\mathbf{x}_{b}, \mathbf{x}_{a}\right)=-\frac{i}{2} \int_{0}^{\infty} d \lambda \int_{-\infty}^{+\infty} \frac{d p_{0}}{2 \pi} e^{-i p_{0}\left(t_{b}-t_{a}\right)+\frac{i \lambda}{2}\left(p_{0}^{2}-m^{2}\right)} \Phi(\lambda) K\left(x_{a}, x_{b}, y_{a}, y_{b} ; \lambda\right)
$$

where $K$ is the propagator of the harmonic oscillator for one charged particle

$$
K\left(x_{a}, x_{b}, y_{a}, y_{b} ; \lambda\right)=\iint D x D y e^{i \int_{0}^{1}\left(\frac{\dot{x}^{2}}{2 \lambda}+\frac{\dot{y}^{2}}{2 \lambda}+G(x \dot{y}-y \dot{x})\right) d \tau}
$$

and the function $\Phi(\lambda)$ reads as

$$
\Phi(\lambda)=\left.\cos (\lambda G) \exp \left(i \gamma \cdot \frac{\partial_{l}}{\partial \theta}\right) \exp \left(\tan (\lambda G) \theta^{1} \theta^{2}\right)\right|_{\theta=0} .
$$

In order to evaluate the propagator $K$, we first decouple $x$ and $y$ by introducing a rotation of coordinates to define new variables $q_{1}$ and $q_{2}$ as

$$
\begin{aligned}
& x=q_{1} \cos (\lambda G \tau)+\kappa q_{2} \sin (\lambda G \tau) \\
& y=-\kappa q_{1} \sin (\lambda G \tau)+q_{2} \cos (\lambda G \tau)
\end{aligned}
$$

where $\kappa=\operatorname{sgn}(G) \neq 0$. Under the above rotation, $K$ can be written in separable form as

$$
K\left(x_{a}, x_{b}, y_{a}, y_{b} ; \lambda\right)=\iint D q_{1} D q_{2} e^{i \int_{0}^{1}\left(\frac{\dot{q}_{1}^{2}}{2 \lambda}+\frac{\dot{q}_{2}^{2}}{2 \lambda}-\frac{\lambda|G|^{2}}{2} q_{1}^{2}-\frac{\lambda|G|^{2}}{2} q_{2}^{2}\right) d \tau}
$$

which represents the kernel of two propagators corresponding to two harmonic oscillators and has well-known form

$$
K\left(x_{a}, x_{b}, y_{a}, y_{b} ; \lambda\right)=\frac{|G|}{2 i \pi \sin (|G| \lambda)} e^{\frac{i|G|}{2 \sin |G| \lambda}\left[\left(\left(x_{b}-x_{a}\right)^{2}+\left(y_{b}-y_{a}\right)^{2}\right) \cos (|G| \lambda)+2 \kappa \sin (|G| \lambda)\left(x_{a} y_{b}-y_{a} x_{b}\right)\right]}
$$


where the following coordinates have been used

$$
\begin{array}{ll}
q_{1 a}=x_{a}, & q_{1 b}=\cos (G \lambda) x_{b}-\kappa \sin (G \lambda) y_{b} \\
q_{2 a}=y_{a}, & q_{2 b}=\kappa \sin (G \lambda) x_{b}+\cos (G \lambda) y_{b} .
\end{array}
$$

To explicitly determine the matrix $\Phi(\lambda)$, let us proceed by using derivation over the variables $\theta$. The calculations performed by adopting the prevailing approach [19] such that acting the operator $\frac{\partial_{l}}{\partial \theta}$ and then replacing $\theta$ by the matrices $\gamma$. We can write the expression of $\Phi(\lambda)$ by using the identities

$$
\begin{aligned}
& \left.\exp \left(i \gamma^{\mu} \frac{\partial_{l}}{\partial \theta^{\mu}}\right) f(\theta)\right|_{\theta=0}=\left.f\left(\frac{\partial_{l}}{\partial \zeta}\right) \exp \left(i \zeta^{\mu} \gamma_{\mu}\right)\right|_{\zeta=0}, \\
& \exp \left(i \zeta^{\mu} \gamma_{\mu}\right)=1+i \zeta^{\mu} \gamma_{\mu}+\frac{1}{2} \zeta^{\mu} \zeta^{\nu} \gamma_{\mu} \gamma_{\nu}+i \zeta^{0} \zeta^{1} \zeta^{2} \gamma_{0} \gamma_{1} \gamma_{2}
\end{aligned}
$$

which are valid for $(2+1)$-dimensional, with

$$
\gamma^{1}=i \sigma_{1}, \quad \gamma^{2}=i \sigma_{2}, \quad \gamma^{0}=\sigma_{3}
$$

where $\zeta^{\mu}$ are odd variables. In this case, we can simplify $\Phi(\lambda)$ as

$$
\Phi(\lambda)=e^{i \kappa|G| \lambda \sigma_{3}}
$$

and therefore (38) becomes

$$
S_{g}^{c}\left(\mathbf{x}_{b}, \mathbf{x}_{a}\right)=-\frac{i}{2} \int_{0}^{\infty} d \lambda \int_{-\infty}^{+\infty} \frac{d p_{0}}{2 \pi} e^{-i p_{0}\left(t_{b}-t_{a}\right)+\frac{i \lambda}{2}\left(p_{0}^{2}-m^{2}\right)} e^{i \kappa|G| \lambda \sigma_{3}} K\left(x_{a}, x_{b}, y_{a}, y_{b} ; \lambda\right)
$$

which can be implemented in (15) to obtain the causal Green function. This task will be done to extract the solutions of the energy spectrum.

\section{$4 \quad$ Energy spectrum}

We would like to determine the eigenvalues and corresponding eigenspinors of our system.To this end, we introduce the polar coordinates $x=r \cos \varphi, y=r \sin \varphi$. Thus, the causal Green function (15) becomes

$$
\begin{aligned}
S^{c}\left(r_{a}, r_{b}, \varphi_{a}, \varphi_{b}, t_{b}, t_{a}\right) & =-\frac{i}{4} \int_{0}^{\infty} d \lambda \int_{-\infty}^{+\infty} \frac{d p_{0}}{2 \pi} e^{-i p_{0}\left(t_{b}-t_{a}\right)+\frac{i \lambda}{2}\left(p_{0}^{2}-m^{2}\right)} \\
& \times\left\{\left(p_{0} \sigma_{3}+\sigma_{1} e^{i \sigma_{3} \varphi_{b}} \frac{\partial}{\partial r_{b}}+\frac{1}{r_{b}} \sigma_{2} e^{i \sigma_{3} \varphi_{b}} \frac{\partial}{\partial \varphi_{b}}-i \kappa|G| \sigma_{2} r_{b} e^{i \sigma_{3} \varphi_{b}}+m\right) e^{i \kappa|G| \lambda \sigma_{3}} K\right. \\
& \left.+e^{i \kappa|G| \lambda \sigma_{3}}\left(p_{0} \sigma_{3}-\sigma_{1} e^{i \sigma_{3} \varphi_{a}} \frac{\partial}{\partial r_{a}}-\frac{1}{r_{a}} \sigma_{2} e^{i \sigma_{3} \varphi_{a}} \frac{\partial}{\partial \varphi_{a}}-i \kappa|G| \sigma_{2} r_{a} e^{i \sigma_{3} \varphi_{a}}+m\right) K\right\}
\end{aligned}
$$

where

$$
K=K\left(r_{a}, r_{b}, \varphi_{a}, \varphi_{b} ; \lambda\right)=\frac{|G|}{2 i \pi \sin (|G| \lambda)} e^{\frac{i|G|}{2 \sin |G| \lambda}\left[\left(r_{b}^{2}+r_{a}^{2}\right) \cos (|G| \lambda)-2 r_{b} r_{a}\left(\cos \left(\varphi_{b}-\varphi_{a}+\kappa|G| \lambda\right)\right)\right]} .
$$

In order to deduce the wave functions, we use the expansion and Hille formulas [22]

$$
\begin{aligned}
& e^{\left(-\frac{i|G|}{\sin |G| \lambda} r_{b} r_{a} \cos \left(\varphi_{b}-\varphi_{a}+\kappa|G| \lambda\right)\right)}=\sum_{l=-\infty}^{+\infty} I_{|l|}\left(-\frac{i|G|}{\sin |G| \lambda} r_{b} r_{a}\right) e^{i l\left(\varphi_{b}-\varphi_{a}+\kappa|G| \lambda\right)} \\
& \frac{(x y t)^{-\frac{\alpha}{2}}}{1-t} \exp \left(-t \frac{x+y}{1-t}\right) I_{\alpha}\left(\frac{2 \sqrt{x y t}}{1-t}\right)=\sum_{n=0}^{\infty} n ! \frac{L_{n}^{\alpha}(x) L_{n}^{\alpha}(y) t^{n}}{\Gamma(n+\alpha+1)}, \quad|t|<1 .
\end{aligned}
$$


By taking $t=e^{-2 i \lambda|G|}, x=|G| r_{b}^{2}, y=|G| r_{a}^{2}$ and $\alpha=|l|$, the propagator $K$ can be expressed as

$$
\begin{aligned}
K\left(r_{a}, r_{b}, \varphi_{a}, \varphi_{b} ; \lambda\right)= & \frac{|G|}{\pi} \sum_{n=0}^{+\infty} \sum_{l=-\infty}^{+\infty} e^{-2 i \lambda|G| n} e^{-i|l||G| \lambda} e^{i l\left(\varphi_{b}-\varphi_{a}+\kappa|G| \lambda\right)} \frac{n !}{\Gamma(n+|l|+1)} \\
& \times e^{-i|G| \lambda} z_{b}^{|l|} z_{a}^{|l|} e^{-\frac{1}{2} z_{b}^{2}} e^{-\frac{1}{2} z_{a}^{2}} L_{n}^{|l|}\left(z_{b}^{2}\right) L_{n}^{|l|}\left(z_{a}^{2}\right)
\end{aligned}
$$

where $z=\sqrt{|G|} r, L_{n}^{|l|}\left(z^{2}\right)$ is a generalized Laguerre polynomial and $|l|$ is an integer number. After inserting (56) into (52) we obtain

$$
\begin{aligned}
S^{c}\left(\mathbf{x}_{b}, \mathbf{x}_{a}\right)= & -\frac{i|G| \sqrt{|G|}}{4 \pi} \sum_{n=0}^{+\infty} \sum_{l=-\infty}^{+\infty} \frac{n !}{(n+|l|) !} \int_{-\infty}^{+\infty} \frac{d p_{0}}{2 \pi} e^{-i p_{0}\left(t_{b}-t_{a}\right)} e^{i l\left(\varphi_{b}-\varphi_{a}\right)} \\
& \times \int_{0}^{\infty} d \lambda e^{\frac{i}{2} \lambda\left(p_{0}^{2}-m^{2}-2|G|[2 n+1+|l|-\kappa l]\right)} \\
& \times\left\{\left(\left(-i \kappa \sigma_{2} z_{b}+\sigma_{1} \frac{\partial}{\partial z_{b}}+i \sigma_{2} \frac{l}{z_{b}}\right) e^{i \sigma_{3} \varphi_{b}}+\frac{m+p_{0} \sigma_{3}}{\sqrt{|G|}}\right) e^{i \kappa|G| \lambda \sigma_{3}}\right. \\
& \left.+e^{i \kappa|G| \lambda \sigma_{3}}\left(e^{-i \sigma_{3} \varphi_{a}}\left(-i \kappa \sigma_{2} z_{a}-\sigma_{1} \frac{\partial}{\partial z_{a}}+i \sigma_{2} \frac{l}{z_{a}}\right)+\frac{m+p_{0} \sigma_{3}}{\sqrt{|G|}}\right)\right\} \\
& \times e^{-\frac{1}{2} z_{b}^{2}} z_{b}^{|l|} L_{n}^{|l|}\left(z_{b}^{2}\right) e^{-\frac{1}{2} z_{a}^{2}} z_{a}^{|l|} L_{n}^{|l|}\left(z_{a}^{2}\right) .
\end{aligned}
$$

Let us introduce the spin operator $\sigma_{3}$ and insert the identity $\sum_{s= \pm 1} \chi_{s} \chi_{s}^{+}=\mathbb{I}$, where $\sigma_{3} \chi_{s}=s \chi_{s}$ and $\chi_{s}^{+} \sigma_{3}=s \chi_{s}^{+}$. We can easily check the relations

$$
\begin{aligned}
& \sigma_{1} \chi_{s}=\chi_{-s}, \quad \sigma_{2} \chi_{s}=i s \chi_{-s} \\
& \chi_{s}^{+} \sigma_{1}=\chi_{-s}^{+}, \quad \chi_{s}^{+} \sigma_{2}=-i s \chi_{-s}^{+}
\end{aligned}
$$

for the vectors

$$
\chi_{+1}=\left(\begin{array}{l}
1 \\
0
\end{array}\right), \quad \chi_{-1}=\left(\begin{array}{l}
0 \\
1
\end{array}\right)
$$

and the identities

$$
\sigma_{2} e^{i \sigma_{3} \varphi}=e^{-i \sigma_{3} \varphi} \sigma_{2}, \quad \sigma_{1} e^{i \sigma_{3} \varphi}=e^{-i \sigma_{3} \varphi} \sigma_{1}
$$

These can be used to write the Green function (57) relative to our particle as

$$
\begin{aligned}
S^{c}\left(\mathbf{x}_{b}, \mathbf{x}_{a}\right)= & -\frac{i|G| \sqrt{|G|}}{4 \pi} \sum_{n=0}^{+\infty} \sum_{l=-\infty}^{+\infty} \sum_{s= \pm 1} \frac{n !}{(n+\mid l) !} \int_{-\infty}^{+\infty} \frac{d p_{0}}{2 \pi} e^{-i p_{0}\left(t_{b}-t_{a}\right)} e^{i l\left(\varphi_{b}-\varphi_{a}\right)} \\
& \times \int_{0}^{\infty} d \lambda e^{\frac{i}{2} \lambda\left(p_{0}^{2}-m^{2}-2|G|[2 n+1+|l|-\kappa(l+s)]\right)} \\
& \times\left\{\left(\left(-i \kappa \sigma_{2} z_{b}+\sigma_{1} \frac{\partial}{\partial z_{b}}+i \sigma_{2} \frac{l}{z_{b}}\right) e^{i s \varphi_{b}}+\frac{m+p_{0} s}{\sqrt{|G|}}\right) \chi_{s} \chi_{s}^{+} e^{i \kappa|G| \lambda s}\right. \\
& \left.+e^{i \kappa|G| \lambda s} \chi_{s} \chi_{s}^{+}\left(e^{-i s \varphi_{a}}\left(-i \kappa \sigma_{2} z_{a}-\sigma_{1} \frac{\partial}{\partial z_{a}}+i \sigma_{2} \frac{l}{z_{a}}\right)+\frac{m+p_{0} s}{\sqrt{|G|}}\right)\right\} \\
& \times e^{-\frac{1}{2} z_{b}^{2}} z_{b}^{|l|} L_{n}^{|l|}\left(z_{b}^{2}\right) e^{-\frac{1}{2} z_{a}^{2}} z_{a}^{|l|} L_{n}^{|l|}\left(z_{a}^{2}\right) .
\end{aligned}
$$

In order to symmetrize the angular part we make the shift

$$
l \rightarrow l-\frac{s+1}{2}
$$


which implies

$$
\begin{aligned}
S^{c}\left(\mathbf{x}_{b}, \mathbf{x}_{a}\right)= & -\frac{i|G| \sqrt{|G|}}{4 \pi} \sum_{n=0}^{\infty} \sum_{l=-\infty}^{+\infty} \sum_{s= \pm 1} \frac{|n| !}{\left(|n|+\left|l-\frac{s+1}{2}\right|\right) !} \int_{-\infty}^{+\infty} \frac{d p_{0}}{2 \pi} e^{-i p_{0}\left(t_{b}-t_{a}\right)} e^{i l\left(\varphi_{b}-\varphi_{a}\right)} e^{-i \frac{\varphi_{b}-\varphi_{a}}{2}} \\
& \times \int_{0}^{\infty} d \lambda e^{\frac{i}{2} \lambda\left(p_{0}^{2}-m^{2}-2|G|\left[2 n+1+\left|l-\frac{s+1}{2}\right|-\kappa\left(l+\frac{s-1}{2}\right)\right]\right)} \\
& \times\left\{\left(\left(\kappa s z_{b}+\frac{\partial}{\partial z_{b}}-s \frac{l-\frac{s+1}{2}}{z_{b}}\right) \chi_{-s} \chi_{s}^{+} e^{i s \frac{\varphi_{b}+\varphi_{a}}{2}}+\frac{m+p_{0} s}{\sqrt{|G|}} e^{-i s \frac{\varphi_{b}-\varphi_{a}}{2}} \chi_{s} \chi_{s}^{+}\right)\right. \\
& \left.+\left(\left(-\kappa s z_{a}-\frac{\partial}{\partial z_{a}}+s \frac{l-\frac{s+1}{2}}{z_{a}}\right) \chi_{s} \chi_{-s}^{+} e^{-i s \frac{\varphi_{b}+\varphi_{a}}{2}}+\chi_{s} \chi_{s}^{+\frac{m+p_{0} s}{\sqrt{|G|}}} e^{-i s \frac{\varphi_{b}-\varphi_{a}}{2}}\right)\right\} \\
& \times e^{-\frac{1}{2} z_{b}^{2}} z_{b}^{\left|l-\frac{s+1}{2}\right|} L_{n}^{\left|l-\frac{s+1}{2}\right|}\left(z_{b}^{2}\right) e^{-\frac{1}{2} z_{a}^{2}} z_{a}^{\left|l-\frac{s+1}{2}\right|} L_{n}^{\left|l-\frac{s+1}{2}\right|}\left(z_{a}^{2}\right) .
\end{aligned}
$$

Introducing

$$
F_{n, l, s}^{\kappa^{\prime}}(z)=\sqrt{\frac{(n) !}{\left(n+\kappa^{\prime}\left(l-\frac{s+1}{2}\right)\right) !}} e^{-\frac{1}{2} z^{2}} z^{\kappa^{\prime}\left(l-\frac{s+1}{2}\right)} L_{n}^{\kappa^{\prime}\left(l-\frac{s+1}{2}\right)}\left(z^{2}\right)
$$

where $\kappa^{\prime}=\operatorname{sgn}\left(l-\frac{s+1}{2}\right)$ and the momentum

$$
\pi_{s}^{\kappa}(z)=\frac{d}{d z}+\kappa s z-s \frac{l-\frac{s+1}{2}}{z}
$$

we write $S^{c}\left(\mathbf{x}_{b}, \mathbf{x}_{a}\right)$ as

$$
\begin{aligned}
S^{c}\left(\mathbf{x}_{b}, \mathbf{x}_{a}\right)= & -\frac{i|G| \sqrt{|G|}}{4 \pi} \sum_{n=0}^{+\infty} \sum_{l=-\infty}^{+\infty} \sum_{s= \pm 1} \int_{-\infty}^{+\infty} \frac{d p_{0}}{2 \pi} e^{-i p_{0}\left(t_{b}-t_{a}\right)} e^{-\frac{1}{2} z_{b}^{2}} e^{-\frac{1}{2} z_{a}^{2}} e^{i l\left(\varphi_{b}-\varphi_{a}\right)} e^{-i \frac{\varphi_{b}-\varphi_{a}}{2}} \\
& \times \sum_{s= \pm 1} \int_{0}^{\infty} d \lambda e^{-\frac{i}{2} \lambda\left(p_{0}^{2}-m^{2}-2|G|\left[2 n+1+\left|l-\frac{s+1}{2}\right|-\kappa\left(l+\frac{s-1}{2}\right)\right]\right)} \\
& \times\left\{\left(e^{i s \frac{\varphi_{b}+\varphi_{a}}{2}} \pi_{s}^{\kappa}\left(z_{b}\right) \chi_{-s} \chi_{s}^{+}+e^{-i s \frac{\varphi_{b}-\varphi_{a}}{2}} \frac{m+p_{0} s}{\sqrt{|G|}} \chi_{s} \chi_{s}^{+}\right) F_{n, l, s}^{\kappa^{\prime}}\left(z_{b}\right) F_{n, l, s}^{\kappa^{\prime}}\left(z_{a}\right)\right. \\
& \left.+\left(-e^{-i s \frac{\varphi_{b}+\varphi_{a}}{2}} \pi_{s}^{\kappa}\left(z_{a}\right) \chi_{s} \chi_{-s}^{+}+e^{-i s \frac{\varphi_{b}-\varphi_{a}}{2}} \frac{m+p_{0} s}{\sqrt{|G|}} \chi_{s} \chi_{s}^{+}\right) F_{n, l, s}^{\kappa^{\prime}}\left(z_{b}\right) F_{n, l, s}^{\kappa^{\prime}}\left(z_{a}\right)\right\} .
\end{aligned}
$$

Integrating over $\lambda$ to get the expression

$$
\left[\frac{i}{2}\left(p_{0}^{2}-m^{2}-2|G|\left[2 n+1+\left|l-\frac{s+1}{2}\right|-\kappa\left(l+\frac{s-1}{2}\right)\right]\right)\right]^{-1}
$$

which has the poles

$$
p_{0}= \pm \sqrt{m^{2}+2|G|\left[2 n+1+\left|l-\frac{s+1}{2}\right|-\kappa\left(l+\frac{s-1}{2}\right)\right]}= \pm E_{n, l, s} .
$$

After using the residue theorem at pole $p_{0}$, we find

$$
\int_{-\infty}^{+\infty} f\left(p_{0}\right) \frac{d p_{0}}{2 \pi} \frac{e^{-i p_{0}\left(t_{b}-t_{a}\right)}}{p_{0}^{2}-E_{n, l, s}^{2}}=-i \sum_{\varepsilon= \pm 1} f\left(\varepsilon E_{n, l, s}\right) \frac{e^{-i \varepsilon E_{n, l, s}\left(t_{b}-t_{a}\right)}}{2 E_{n, l, s}} \Theta\left(\varepsilon\left(t_{b}-t_{a}\right)\right)
$$


where $\Theta(x)$ is the Heaviside step function. Since $\varepsilon= \pm 1$ and $s= \pm 1$, one can easily check the identity $\sum_{s= \pm 1} f_{s} \sum_{\varepsilon= \pm 1} g_{\varepsilon}=\sum_{s= \pm 1} f_{s}\left(g_{s}+g_{-s}\right)$. After rearranging different terms, we obtain

$$
\begin{aligned}
& S^{c}\left(\mathbf{x}_{b}, \mathbf{x}_{a}\right)=\frac{i}{2 \pi} \sqrt{|G|^{3}} \sum_{n=0}^{+\infty} \sum_{l=-\infty}^{+\infty} \sum_{s= \pm 1} \frac{1}{2 E_{n, l, s}} e^{i(l-1 / 2)\left(\varphi_{b}-\varphi_{a}\right)} \\
& \times\left\{e ^ { - i s E _ { n , l , s } ( t _ { b } - t _ { a } ) } \Theta ( s ( t _ { b } - t _ { a } ) ) \left[\left(e^{i s \frac{\varphi_{b}+\varphi_{a}}{2}} \pi_{s}^{\kappa}\left(z_{b}\right) \chi_{-s} \chi_{s}^{+}+e^{-i s \frac{\varphi_{b}-\varphi_{a}}{2}} \frac{m+E_{n, l, s}}{\sqrt{|G|}} \chi_{s} \chi_{s}^{+}\right)\right.\right. \\
& \left.-\left(e^{-i s \frac{\varphi_{b}+\varphi_{a}}{2}} \pi_{s}^{\kappa}\left(z_{a}\right) \chi_{s} \chi_{-s}^{+}-e^{-i s \frac{\varphi_{b}-\varphi_{a}}{2}} \frac{m+E_{n, l, s}}{\sqrt{|G|}} \chi_{s} \chi_{s}^{+}\right)\right] \\
& +e^{i s E_{n, l, s}\left(t_{b}-t_{a}\right)} \Theta\left(-s\left(t_{b}-t_{a}\right)\right)\left[\left(e^{i s \frac{\varphi_{b}+\varphi_{a}}{2}} \pi_{s}^{\kappa}\left(z_{b}\right) \chi_{-s} \chi_{s}^{+}+e^{-i s \frac{\varphi_{b}-\varphi_{a}}{2}} \frac{m-E_{n, l, s}}{\sqrt{|G|}} \chi_{s} \chi_{s}^{+}\right)\right. \\
& \left.\left.-\left(e^{-i s \frac{\varphi_{b}+\varphi_{a}}{2}} \pi_{s}^{\kappa}\left(z_{a}\right) \chi_{s} \chi_{-s}^{+}-e^{-i s \frac{\varphi_{b}-\varphi_{a}}{2}} \frac{m-E_{n, l, s}}{\sqrt{|G|}} \chi_{s} \chi_{s}^{+}\right)\right]\right\} F_{n, l, s}^{\kappa^{\prime}}\left(z_{b}\right) F_{n, l, s}^{\kappa^{\prime}}\left(z_{a}\right) .
\end{aligned}
$$

To go further, we proceed by using the following mapping

$$
s \rightarrow s^{\prime}=-s, \quad n \rightarrow n^{\prime}=n-\frac{\kappa^{\prime}+\kappa}{2} s= \begin{cases}n-\kappa s, & \text { if } \kappa^{\prime}=\kappa \text { or } \kappa^{\prime}=0 \\ n, & \text { if } \kappa^{\prime}=-\kappa\end{cases}
$$

only for terms containing $\Theta\left(-s\left(t_{b}-t_{a}\right)\right)$ in (71) to the dummy variables $n$ and $s$, which does not alter the integer nature of $n$. By taking into consideration the energy invariance under (72), the Green function takes the following form

$$
\begin{aligned}
S^{c}\left(\mathbf{x}_{b}, \mathbf{x}_{a}\right)= & \frac{i}{2 \pi} \sqrt{|G|^{3}} \sum_{n=0}^{+\infty} \sum_{l=-\infty}^{+\infty} \sum_{s= \pm 1} \frac{1}{2 E_{n, l, s}} e^{i(l-1 / 2)\left(\varphi_{b}-\varphi_{a}\right)} e^{-i s E_{n, l, s}\left(t_{b}-t_{a}\right)} \Theta\left(s\left(t_{b}-t_{a}\right)\right) \\
& \times\left\{\left[\left(e^{i s \frac{\varphi_{b}+\varphi_{a}}{2}} \pi_{s}^{\kappa}\left(z_{b}\right) \chi_{s^{\prime}} \chi_{s}^{+}+e^{-i s \frac{\varphi_{b}-\varphi_{a}}{2}} \frac{m+E_{n, l, s}}{\sqrt{|G|}} \chi_{s} \chi_{s}^{+}\right)\right.\right. \\
& \left.+\left(-e^{-i s \frac{\varphi_{b}+\varphi_{a}}{2}} \pi_{s}^{\kappa}\left(z_{a}\right) \chi_{s} \chi_{s^{\prime}}^{+}+e^{-i s \frac{\varphi_{b}-\varphi_{a}}{2}} \frac{m+E_{n, l, s}}{\sqrt{|G|}} \chi_{s} \chi_{s}^{+}\right)\right] F_{n, l, s}^{\kappa^{\prime}}\left(z_{b}\right) F_{n, l, s}^{\kappa^{\prime}}\left(z_{a}\right) \\
& +\left[\left(e^{-i \frac{\varphi_{b}+\varphi_{a}}{2}} \pi_{s^{\prime}}^{\kappa}\left(z_{b}\right) \chi_{s} \chi_{s^{\prime}}^{+}+e^{i s \frac{\varphi_{b}-\varphi_{a}}{2}} \frac{m-E_{n, l, s}}{\sqrt{|G|}} \chi_{s^{\prime}} \chi_{s^{\prime}}^{+}\right)\right. \\
& \left.\left.+\left(-e^{i s \frac{\varphi_{b}+\varphi_{a}}{2}} \pi_{s^{\prime}}^{\kappa}\left(z_{a}\right) \chi_{s^{\prime}} \chi_{s}^{+}+e^{i s \frac{\varphi_{b}-\varphi_{a}}{2}} \frac{m-E_{n, l, s}}{\sqrt{|G|}} \chi_{s^{\prime}} \chi_{s^{\prime}}^{+}\right)\right] F_{n^{\prime}, l, s^{\prime}}^{\kappa^{\prime}}\left(z_{b}\right) F_{n^{\prime}, l, s^{\prime}}^{\kappa^{\prime}}\left(z_{a}\right)\right\}
\end{aligned}
$$

By virtue of Rodrigues' formula

$$
L_{n}^{\alpha}(x)=\frac{1}{n !} e^{x} x^{-\alpha} \frac{d^{n}}{d x^{n}}\left(e^{-x} x^{n+\alpha}\right)
$$

we deduce after verification for all numbers $\left(\kappa, \kappa^{\prime}, s\right)$ the interesting properties

$$
\begin{aligned}
& \pi_{s}^{\kappa}(z) F_{n, l, s}^{\kappa^{\prime}}(z)=-s \sqrt{\frac{E_{n, l, s}^{2}-m^{2}}{|G|}} F_{n^{\prime}, l, s^{\prime}}^{\kappa^{\prime}}(z) \\
& \pi_{s^{\prime}}^{\kappa}(z) F_{n^{\prime}, l, s^{\prime}}^{\kappa^{\prime}}(z)=s \sqrt{\frac{E_{n, l, s}^{2}-m^{2}}{|G|}} F_{n, l, s}^{\kappa^{\prime}}(z) .
\end{aligned}
$$


These give

$$
\begin{aligned}
S^{c}\left(\mathbf{x}_{b}, \mathbf{x}_{a}\right)= & i \frac{|G|}{\pi} \sum_{n=0}^{+\infty} \sum_{l=-\infty}^{+\infty} \sum_{s= \pm 1} \frac{1}{2 E_{n, l, s}} e^{i(l-1 / 2)\left(\varphi_{b}-\varphi_{a}\right)} e^{-i s E_{n, l, s}\left(t_{b}-t_{a}\right)} \Theta\left(s\left(t_{b}-t_{a}\right)\right) \\
& \times\left\{e^{-i s \frac{\varphi_{b}-\varphi_{a}}{2}}\left(E_{n, l, s}+m\right) F_{n, l, s}^{\kappa^{\prime}}\left(z_{b}\right) F_{n, l, s}^{\kappa^{\prime}}\left(z_{a}\right) \chi_{s} \chi_{s}^{+}\right. \\
& +e^{i s \frac{\varphi_{b}-\varphi_{a}}{2}}\left(E_{n, l, s}-m\right) F_{n^{\prime}, l, s^{\prime}}^{\kappa^{\prime}}\left(z_{b}\right) F_{n^{\prime}, l, s^{\prime}}^{\kappa^{\prime}}\left(z_{a}\right) \chi_{s^{\prime}} \chi_{s^{\prime}}^{+} \\
& -s \sqrt{E_{n, l, s}^{2}-m^{2}}\left[e^{-i s \frac{\varphi_{b}+\varphi_{a}}{2}} F_{n, l, s}^{\kappa^{\prime}}\left(z_{b}\right) F_{n^{\prime}, l, s^{\prime}}^{\kappa^{\prime}}\left(z_{a}\right) \chi_{s} \chi_{s^{\prime}}^{+}\right. \\
& \left.\left.+e^{i \frac{\varphi_{b}+\varphi_{a}}{2}} F_{n^{\prime}, l, s^{\prime}}^{\kappa^{\prime}}\left(z_{b}\right) F_{n, l, s}^{\kappa^{\prime}}\left(z_{a}\right) \chi_{s^{\prime}} \chi_{s}^{+}\right]\right\} s \sigma_{3}
\end{aligned}
$$

which can be simplified to end up with

$$
S^{c}\left(\mathbf{x}_{b}, \mathbf{x}_{a}\right)=i \sum_{n=0}^{+\infty} \sum_{l=-\infty}^{+\infty} \sum_{s= \pm 1} \Psi_{n, l, s}^{\kappa, \kappa^{\prime}}\left(r_{b}, \varphi_{b} ; t_{b}\right)\left(\Psi_{n, l, s}^{\kappa, \kappa^{\prime}}\left(r_{a}, \varphi_{a} ; t_{a}\right)\right)^{+} \sigma_{3} s \Theta\left(s\left(t_{b}-t_{a}\right)\right)
$$

where the normalized wave functions are given by

$$
\Psi_{n, l, s}^{\kappa, \kappa^{\prime}}(r, \varphi ; t)=\sqrt{\frac{|G|}{\pi}} e^{-i \mathcal{E}_{n, l, s} t} e^{i l \varphi} e^{-i \frac{\sigma_{3}+1}{2} \varphi}\left[\sqrt{\frac{\mathcal{E}_{n, l, s}+s m}{2 \mathcal{E}_{n, l, s}}} F_{n, l, s}^{\kappa^{\prime}}(z) \chi_{s}-s \sqrt{\frac{\mathcal{E}_{n, l, s}-s m}{2 \mathcal{E}_{n, l, s}}} F_{n-\frac{\kappa^{\prime}+\kappa}{2} s, l,-s}^{\kappa^{\prime}}(z) \chi_{-s}\right]
$$

and the corresponding eigenvalues take the form

$$
\mathcal{E}_{n, l, s}=s E_{n, l, s}=s \sqrt{m^{2}+2|G|\left[2 n+1+\kappa^{\prime}\left(l-\frac{s+1}{2}\right)-\kappa\left(l+\frac{s-1}{2}\right)\right]} .
$$

In compact form, we have the solutions of the energy spectrum

$$
\begin{aligned}
\Psi_{n, l, s}(r, \varphi ; t)= & \sqrt{\frac{|G|}{\pi}} e^{-i s \mathcal{E}_{n, l, s} t} e^{i l \varphi} e^{-i \frac{\sigma_{3}+1}{2} \varphi} \\
& \times\left[\sqrt{\frac{\mathcal{E}_{n, l, s}+s m}{2 \mathcal{E}_{n, l, s}}} F_{n, l, s}(z) \chi_{s}+\sqrt{\frac{|G|}{2 \mathcal{E}_{n, l, s}\left(\mathcal{E}_{n, l, s}+s m\right)}} \pi_{s}(z) F_{n, l, s}(z) \chi_{-s}\right] \\
\mathcal{E}_{n, l, s} & =s \sqrt{m^{2}+2|G|\left[2 n+1+\left|l-\frac{s+1}{2}\right|-\frac{G}{|G|}\left(l+\frac{s-1}{2}\right)\right]}
\end{aligned}
$$

with the quantities $\kappa=\operatorname{sgn}(G)=\frac{G}{|G|} \neq 0, \kappa^{\prime}=\operatorname{sgn}\left(l-\frac{s+1}{2}\right), s=\operatorname{sgn}\left(\mathcal{E}_{n, l, s}\right)$ and the variable $z=\sqrt{|G|}$. It is interesting to note that our obtained energy spectrum is completely in agreement with that derived quantum mechanically in [15]

Note that by putting $\omega=0$, which gives $G=-m \omega_{c}$, and making the substitution $l \longrightarrow l+\frac{s+1}{2}$ in (82) we end up with the energy

$$
E_{s= \pm 1}^{2}=m^{2}+2 m \omega_{c}[2 n+1+|l|+l+s] .
$$

This can be compared to the energy for a massive fermion in a constant magnetic field in 3+1dimensional space-time [24]. It is given by

$$
E_{\lambda= \pm 1}^{2}-p_{z}^{2}=m^{2}+2 m \kappa[2 n+|l|+l-\lambda+1]
$$

which shows that it is in agreement with our derived result (83). 


\section{$5 \quad$ Hidden symmetries}

We show that there are nontrivial hidden symmetries in our solutions of the energy spectrum. As far as the eigenvalues are concerned, we notice that by considering three configurations of quantum number $(n, l, s)$, the energy remains invariant absolutely. These are listed in the following table

\begin{tabular}{|c|c|c|c|}
\hline \hline Sign $s$ & Quantum number $l$ & Quantum number $n$ & Energy $\mathcal{E}_{n, l, s}$ \\
\hline \hline$s \longrightarrow-s$ & $l \longrightarrow l-s$ & $n \longrightarrow n-\kappa s$ & $\mathcal{E}_{n-\kappa s, l-s,-s}=-\mathcal{E}_{n, l, s}$ \\
\hline \hline$s \longrightarrow-s$ & $l \longrightarrow l+s$ & $n \longrightarrow n-\kappa^{\prime} s$ & $\mathcal{E}_{n-\kappa^{\prime} s, l+s,-s}=-\mathcal{E}_{n, l, s}$ \\
\hline \hline$s \longrightarrow-s$ & $l \longrightarrow l$ & $n \longrightarrow n-\frac{\kappa^{\prime}+\kappa}{2} s$ & $\mathcal{E}_{n-\frac{\kappa^{\prime} \kappa}{2}} s, l,-s$ \\
2 & $-\mathcal{E}_{n, l, s}$ \\
\hline \hline
\end{tabular}

Table 1: Table summarizes different symmetries of our system according to changes in terms of the quantum numbers $(n, l, s)$.

Furthermore, similar attention reed to be paid to the eigenspinors $\Psi_{n, l, s}(r, \varphi ; t)$ and underline their basic features. Let us focus particularly on the last configuration and express $\Psi_{n, l, s}(r, \varphi ; t)$ as

$$
\Psi_{n, l, s}(r, \varphi ; t)=u_{n, l, s}(r, \varphi ; t) \chi_{s}+v_{n, l, s}(r, \varphi ; t) \chi_{-s}
$$

where $u_{n, l, s}$ and $v_{n, l, s}$ are two-component defined by

$$
\begin{aligned}
& u_{n, l, s}(r, \varphi ; t)=e^{-i \mathcal{E}_{n, l, s} t} \sqrt{\frac{|G|}{\pi}} e^{i l \varphi} e^{-i \frac{s+1}{2} \varphi} \sqrt{\frac{\mathcal{E}_{n, l, s}+s m}{2 \mathcal{E}_{n, l, s}}} F_{n, l, s}^{\kappa^{\prime}}(z) \\
& v_{n, l, s}(r, \varphi ; t)=-s e^{-i \mathcal{E}_{n, l, s} t} \sqrt{\frac{|G|}{\pi}} e^{i l \varphi} e^{-i \frac{-s+1}{2} \varphi} \sqrt{\frac{\mathcal{E}_{n, l, s}-s m}{2 \mathcal{E}_{n, l, s}}} F_{n-\frac{\kappa^{\prime}+\kappa}{2} s, l,-s}^{\kappa^{\prime}}(z) .
\end{aligned}
$$

Now using the third configuration in Table 1 to show there is a relation between $v_{n, l, s}$ and $u_{n, l, s}$. This is given by

$$
v_{n-\frac{\kappa^{\prime}+\kappa}{2} s, l,-s}(r, \varphi ;-t)=s \sqrt{\frac{\mathcal{E}_{n, l, s}-s m}{\mathcal{E}_{n, l, s}+s m}} u_{n, l, s}(r, \varphi ; t)
$$

which is in agreement with [23]. According to the above results, we conclude that the obtained energy spectrum is invariant with respect to the symmetries listed above. This invariance can be used to deal with some issues related to the present system.

\section{Density of current}

Let us consider the density of current $\vec{J}$ for our system and investigate its basic features. Indeed, from our results we can end up with the form

$$
\vec{J}=i\left\langle\sigma_{3} \vec{\sigma}\right\rangle
$$

For this calculation, we use the spinor wavefunction obtained above. This gives a null value in Cartesian coordinates, i.e. $J_{x}=J_{y}=0$, which of course, is expected since there is no net charge drift in our system. 
As a reassuring exercise, we calculate the same current $\vec{J}$ but in cylindrical coordinates. Doing this process to obtain two components

$$
J_{r}=\vec{J} . \hat{r}=i\left\langle\sigma_{3} \vec{\sigma} \cdot \hat{r}\right\rangle, \quad J_{\theta}=\vec{J} \cdot \hat{\theta}=i\left\langle\sigma_{3} \vec{\sigma} \cdot \hat{\theta}\right\rangle .
$$

We can show that the radial component is null, i.e. $\bar{J}_{r}^{n, l, s}=0$. For the angular one, we use the definition to obtain

$$
\bar{J}_{\varphi}^{n, l, s}=\int_{0}^{+\infty} \int_{0}^{2 \pi} \Psi_{n, l, s}^{+}(r, \varphi) \sigma_{1} e^{i \sigma_{3} \varphi} \Psi_{n, l, s}(r, \varphi) r d r d \varphi
$$

which can be written as

$$
\begin{aligned}
\bar{J}_{\varphi}^{n, l, s} & =2|G| \sqrt{\frac{|G|}{\mathcal{E}_{n, l, s}^{2}}} \int_{0}^{+\infty} F_{n, l, s}(z) \pi_{s}^{\kappa}(z) F_{n, l, s}(z) r d r \\
& =2|G| \sqrt{\frac{|G|}{\mathcal{E}_{n, l, s}^{2}}} \int_{0}^{+\infty} F_{n, l, s}(z)\left(\frac{d}{d z}+\kappa s z-s \frac{l-\frac{s+1}{2}}{z}\right) F_{n, l, s}(z) r d r .
\end{aligned}
$$

Using the relation [21]

$$
\int_{0}^{+\infty} x^{\alpha-1} e^{-x} L_{m}^{\gamma}(x) L_{n}^{\lambda}(x) d x=\frac{(1+\gamma)_{m}(\lambda-\alpha+1)_{n} \Gamma(\alpha)}{m ! n !}{ }_{3} F_{2}(-m, \alpha, \alpha-\lambda ; 1+\gamma, \alpha-\lambda-n ; 1)
$$

for $\operatorname{Re}(\alpha)>0$, the well-known recurrence and derivative properties of generalized Laguerre functions to obtain the final form for $(92)$

$$
\begin{aligned}
\bar{J}_{\varphi}^{n, l, s}= & 2 s \frac{n ! \sqrt{|G|}}{\left(n+\left|l-\frac{s+1}{2}\right|\right) ! \mathcal{E}_{n, l, s}}\left(\left|l-\frac{s+1}{2}\right|-s\left(l-\frac{s+1}{2}\right)\right) \frac{\left(1+\left|l-\frac{s+1}{2}\right|\right)_{n}\left(\frac{1}{2}\right)_{n} \Gamma\left(\left|l-\frac{s+1}{2}\right|+\frac{1}{2}\right)}{(n !)^{2}} \\
& \times{ }_{3} F_{2}\left(-n,\left|l-\frac{s+1}{2}\right|+\frac{1}{2}, \frac{1}{2} ; 1+\left|l-\frac{s+1}{2}\right|, \frac{1}{2}-n ; 1\right) \\
& -4 s \frac{n ! \sqrt{|G|}}{\left(n+\left|l-\frac{s+1}{2}\right|\right) ! \mathcal{E}_{n, l, s}} \frac{\left(2+\left|l-\frac{s+1}{2}\right|\right)_{n-1}\left(-\frac{1}{2}\right)_{n} \Gamma\left(\left|l-\frac{s+1}{2}\right|+\frac{3}{2}\right)}{(n-1) ! n !} \\
& \times{ }_{3} F_{2}\left(-n+1,\left|l-\frac{s+1}{2}\right|+\frac{3}{2}, \frac{3}{2} ; 2+\left|l-\frac{s+1}{2}\right|, \frac{3}{2}-n ; 1\right) \\
& +2 s \frac{n ! \sqrt{|G|}}{\left(n+\left|l-\frac{s+1}{2}\right|\right) ! \mathcal{E}_{n, l, s}}\left(\frac{G}{|G|} s-1\right) \frac{\left(1+\left|l-\frac{s+1}{2}\right|\right)_{n}\left(-\frac{1}{2}\right)_{n} \Gamma\left(\left|l-\frac{s+1}{2}\right|+\frac{3}{2}\right)}{(n !)^{2}} \\
& \times{ }_{3} F_{2}\left(-n,\left|l-\frac{s+1}{2}\right|+\frac{3}{2}, \frac{3}{2} ; 1+\left|l-\frac{s+1}{2}\right|, \frac{3}{2}-n ; 1\right)
\end{aligned}
$$

where ${ }_{3} F_{2}$ is the generalized hypergeometric function. This expression can be worked under different assumptions to end up with a simple form and therefore make link with some physical phenomena. Let us illustrate (94) by summarizing some simple forms in Table 2

\begin{tabular}{|c|c|c|}
\hline \hline Configuration & Quantum numbers $n, l, s$ & Angular current $\bar{J}_{\varphi}^{n, l, s}$ \\
\hline \hline 1 & $n=0, l=0, s=+1$ & $\frac{1}{4}(1+3 \kappa) \sqrt{\frac{\pi|G|}{m^{2}+4|G|}}$ \\
\hline \hline 2 & $n=0, l=0, s=-1$ & $-\frac{1}{2}(1+\kappa) \sqrt{\frac{\pi|G|}{m^{2}+2|G|(1+\kappa)}}$ \\
\hline \hline 3 & $n=0, l=1, s=+1$ & $\frac{1}{4}(1-3 \kappa) \sqrt{\frac{\pi|G|}{m^{2}+4|G|}}$ \\
\hline \hline 4 & $n=0, l=1, s=-1$ & $-\frac{1}{2}(1-\kappa) \sqrt{\frac{\pi|G|}{m^{2}+2|G|(1-\kappa)}}$ \\
\hline \hline
\end{tabular}


Table 2: Table illustrates some examples of the angular current for some quantum numbers values $(n, l, s)$.

From Table 2, we can immediately realize that there are some symmetries. Indeed, for the configurations $(1,3)$ one can establish the relation

$$
\bar{J}_{\varphi}^{0,0,+1}(\kappa)=\bar{J}_{\varphi}^{0,1,+1}(-\kappa)
$$

and the same between $(2,4)$

$$
\bar{J}_{\varphi}^{0,0,-1}(\kappa)=\bar{J}_{\varphi}^{0,1,-1}(-\kappa)
$$

under the change $\kappa \longrightarrow-\kappa$, with the parameter $\kappa=\operatorname{sgn}(G)=\frac{G}{|G|} \neq 0$. We expect to have other symmetries can be found by choosing new configurations of the three quantum numbers. This is interesting and could be used to formulate a theory to describe low dimensional systems without having external excitations.

\section{$7 \quad$ Nonrelativistic limit}

To recover the nonrelativistic limit, we use the standard process. Indeed, by requiring the limit $m \longrightarrow \infty$ in the obtained solutions of the energy spectrum, one can see

$$
\begin{gathered}
\mathcal{E}_{n, l, s} \longrightarrow s m\left(1+\frac{|G|}{m^{2}}\left[2 n+1+\left|l-\frac{s+1}{2}\right|-\frac{G}{|G|}\left(l+\frac{s-1}{2}\right)\right]\right) \\
\sqrt{\frac{\mathcal{E}_{n, l, s}+s m}{2 \mathcal{E}_{n, l, s}}} \longrightarrow 1 \\
\sqrt{\frac{\mathcal{E}_{n, l, s}-s m}{2 \mathcal{E}_{n, l, s}}} \longrightarrow 0 .
\end{gathered}
$$

These allow the Green function to behave as

$$
\begin{aligned}
S^{c}\left(\mathbf{x}_{b}, \mathbf{x}_{a}\right) \longrightarrow & i \frac{|G|}{\pi} \sum_{n=0}^{+\infty} \sum_{l=-\infty}^{+\infty} \sum_{s= \pm 1} e^{i(l-(1+s) / 2)\left(\varphi_{b}-\varphi_{a}\right)} e^{-i\left(s m+s \frac{|G|}{m}\left[2 n+1+\left|l-\frac{s+1}{2}\right|-\frac{G}{|G|}\left(l+\frac{s-1}{2}\right)\right]\right)\left(t_{b}-t_{a}\right)} \\
& \times \Theta\left(s\left(t_{b}-t_{a}\right)\right) F_{n, l, s}^{\kappa^{\prime}}\left(z_{b}\right) F_{n, l, s}^{\kappa^{\prime}}\left(z_{a}\right) \chi_{s} \chi_{s}^{+} .
\end{aligned}
$$

Now taking $l \longrightarrow l+\frac{s+1}{2}$ to write (100) as

$$
\begin{aligned}
S^{c}\left(\mathbf{x}_{b}, \mathbf{x}_{a}\right) \longrightarrow & i \frac{|G|}{\pi} \sum_{n=0}^{+\infty} \sum_{l=-\infty}^{+\infty} e^{i l\left(\varphi_{b}-\varphi_{a}\right)} e^{-i\left(m+\frac{|G|}{m}\left[2 n+1+|l|-\frac{G}{|G|}(l+1)\right]\right)\left(t_{b}-t_{a}\right)} \Theta\left(\left(t_{b}-t_{a}\right)\right) \\
& \times F_{n, l}\left(z_{b}\right) F_{n, l}\left(z_{a}\right) \chi_{+1} \chi_{+1}^{+} \\
& +i \frac{|G|}{\pi} \sum_{n=0}^{+\infty} \sum_{l=-\infty}^{+\infty} e^{i l\left(\varphi_{b}-\varphi_{a}\right)} e^{i\left(m+\frac{|G|}{m}\left[2 n+1+|l|-\frac{G}{|G|}(l-1)\right]\right)\left(t_{b}-t_{a}\right)} \Theta\left(-\left(t_{b}-t_{a}\right)\right) \\
& \times F_{n, l}\left(z_{b}\right) F_{n, l}\left(z_{a}\right) \chi_{-1} \chi_{-1}^{+}
\end{aligned}
$$

and therefore to end up with usual eigenfunctions for the nonrelativistic problem

$$
\Psi_{n, l, s}(r, \varphi ; t) \rightarrow e^{-i\left(m+\frac{|G|}{m}\left[2 n+1+|l|-\frac{G}{|G|}(l+1)\right]\right) t}\left(\begin{array}{c}
\Psi^{N R}(r, \varphi) \\
0
\end{array}\right)
$$


or in the form

$$
\Psi_{n, l, s}(r, \varphi ; t) \rightarrow e^{+i\left(m+\frac{|G|}{m}\left[2 n+1+|l|-\frac{G}{|G|}(l-1)\right]\right) t}\left(\begin{array}{c}
0 \\
\Psi^{N R}(r, \varphi)
\end{array}\right)
$$

where $\Psi^{N R}(r, \varphi)$ are the nonrelativistic functions defined by

$$
\Psi^{N R}(r, \varphi)=\sqrt{\frac{|G| n !}{\pi(n+|l|) !}} e^{i l \varphi}(\sqrt{|G| r})^{|l|} e^{-\frac{1}{2}|G| r^{2}} L_{n}^{|l|}\left(|G| r^{2}\right)
$$

and the corresponding nonrelativistic energy is

$$
\mathcal{E}^{N R}=\frac{|G|}{m}\left[2 n+1+|l|-\frac{G}{|G|}(l \pm 1)\right]=\frac{\mathcal{E}_{n, l, s}^{2}-m^{2}}{2 m} .
$$

The same results can be found for one-relativistic particle living on the plane $(x ; y)$ in presence of a perpendicular magnetic field $B$ described by the Pauli-Schrödinger Hamiltonian

$$
H^{P S}=\frac{1}{2 m}[\vec{\sigma} \cdot(\vec{P}-e \vec{A})]^{2}
$$

in the symmetric gauge $\vec{A}=\frac{B}{2}(-y, x)$, more detail can be found in [6]. This tells us that our findings are interesting and allow to recover the well-known results for the present problem.

\section{Completeness}

It is interesting to examine the completeness relation associated to our solutions of the energy spectrum. Indeed, the closure relation obeyed by eigenfunctions is given by

$$
\sum_{n=0}^{+\infty} \sum_{l=-\infty}^{+\infty} \sum_{s= \pm 1} \Psi_{n, l, s}^{\kappa, \kappa^{\prime}}\left(\mathbf{x}_{b}\right)\left(\Psi_{n, l, s}^{\kappa, \kappa^{\prime}}\left(\mathbf{x}_{a}\right)\right)^{+}=\mathbb{I}_{2 \times 2} \delta\left(\mathbf{x}_{b}-\mathbf{x}_{a}\right) .
$$

Introducing

$$
\begin{aligned}
& I\left(\mathbf{x}_{b}, \mathbf{x}_{a}\right)=\sum_{n=0}^{+\infty} \sum_{l=-\infty}^{+\infty} \sum_{s= \pm 1} \Psi_{n, l, s}^{\kappa, \kappa^{\prime}}\left(r_{b}, \varphi_{b}\right)\left(\Psi_{n, l, s}^{\kappa, \kappa^{\prime}}\left(r_{a}, \varphi_{a}\right)\right)^{+} \\
& =\sqrt{\frac{|G|}{\pi}} e^{i l \varphi_{b}}\left[\sqrt{\frac{\mathcal{E}_{n, l, s}+s m}{2 \mathcal{E}_{n, l, s}}} F_{n, l, s}^{\kappa^{\prime}}(z) \chi_{s}-s \sqrt{\frac{\mathcal{E}_{n, l, s}-s m}{2 \mathcal{E}_{n, l, s}}} F_{n-\frac{\kappa^{\prime}+\kappa}{2} s, l,-s}^{\kappa^{\prime}}(z) \chi_{-s}\right] \\
& \times \sqrt{\frac{|G|}{\pi}}\left[\sqrt{\frac{\mathcal{E}_{n, l, s}+s m}{2 \mathcal{E}_{n, l, s}}} F_{n, l, s}^{\kappa^{\prime}}(z) \chi_{s}^{+}-s \sqrt{\frac{\mathcal{E}_{n, l, s}-s m}{2 \mathcal{E}_{n, l, s}}} F_{n-\frac{\kappa^{\prime}+\kappa}{2} s, l,-s}^{\kappa^{\prime}}(z) \chi_{-s}^{+}\right] \\
& =\frac{|G|}{\pi} \sum_{n=0}^{+\infty} \sum_{l=-\infty}^{+\infty} \sum_{s= \pm 1}\left\{\frac{\mathcal{E}_{n, l, s}+s m}{2 \mathcal{E}_{n, l, s}} F_{n, l, s}^{\kappa^{\prime}}\left(z_{b}\right) F_{n, l, s}^{\kappa^{\prime}}\left(z_{a}\right) e^{-i \frac{s+1}{2} \varphi_{b}} e^{i \frac{s+1}{2} \varphi_{a}} \chi_{s} \chi_{s}^{+}\right. \\
& +\frac{\mathcal{E}_{n, l, s}-s m}{2 \mathcal{E}_{n, l, s}} F_{n-\frac{\kappa^{\prime}+\kappa}{2} s, l,-s}^{\kappa^{\prime}}\left(z_{b}\right) F_{n-\frac{\kappa^{\prime}+\kappa}{2}}^{\kappa^{\prime}, l,-s}\left(z_{a}\right) e^{-i \frac{-s+1}{2} \varphi_{b}} e^{i \frac{-s+1}{2} \varphi_{a}} \chi_{-s} \chi_{-s}^{+} \\
& -s \frac{\mathcal{E}_{n, l, s}-s m}{2 \mathcal{E}_{n, l, s}} \frac{\mathcal{E}_{n, l, s}+s m}{2 \mathcal{E}_{n, l, s}} F_{n-\frac{\kappa^{\prime}+\kappa}{2} s, l,-s}^{\kappa^{\prime}}\left(z_{b}\right) F_{n, l, s}^{\kappa^{\prime}}\left(z_{a}\right) e^{-i \frac{-s+1}{2} \varphi_{b}} e^{i \frac{s+1}{2} \varphi_{a}} \chi_{-s} \chi_{s}^{+} \\
& \left.-s \frac{\mathcal{E}_{n, l, s}-s m}{2 \mathcal{E}_{n, l, s}} \frac{\mathcal{E}_{n, l, s}+s m}{2 \mathcal{E}_{n, l, s}} F_{n-\frac{\kappa^{\prime}+\kappa}{2} s, l,-s}^{\kappa^{\prime}}\left(z_{a}\right) F_{n, l, s}^{\kappa^{\prime}}\left(z_{b}\right) e^{-i \frac{s+1}{2} \varphi_{b}} e^{i \frac{-s+1}{2} \varphi_{a}} \chi_{s} \chi_{-s}^{+}\right\} .
\end{aligned}
$$


Taking into account of the symmetry properties discussed in section 5 , in particular the changes $s \rightarrow-s$ and $n \rightarrow n-\frac{\kappa^{\prime}+\kappa}{2} s$, one can simplify $I\left(\mathbf{x}_{b}, \mathbf{x}_{a}\right)$ as

$$
\begin{aligned}
I\left(\mathbf{x}_{b}, \mathbf{x}_{a}\right)= & \frac{|G|}{\pi} \sum_{n=0}^{+\infty} \sum_{l=-\infty}^{+\infty} \sum_{s= \pm 1} F_{n, l, s}^{\kappa^{\prime}}\left(z_{b}\right) F_{n, l, s}^{\kappa^{\prime}}\left(z_{a}\right) e^{-i \frac{s+1}{2} \varphi_{b}} e^{i \frac{s+1}{2} \varphi_{a}} \chi_{s} \chi_{s}^{+} \\
= & \frac{|G|}{\pi} \sum_{n=0}^{+\infty} \sum_{l=-\infty}^{+\infty} \sum_{s= \pm 1}\left\{\frac{(n) !}{\left(n+\kappa^{\prime}\left(l-\frac{s+1}{2}\right)\right) !} e^{-\frac{1}{2} z_{b}^{2}} z_{b}^{\kappa^{\prime}\left(l-\frac{s+1}{2}\right)} L_{n}^{\kappa^{\prime}\left(l-\frac{s+1}{2}\right)}\left(z_{b}^{2}\right)\right. \\
& \left.\times e^{-\frac{1}{2} z_{a}^{2}} z_{a}^{\kappa^{\prime}\left(l-\frac{s+1}{2}\right)} L_{n}^{\kappa^{\prime}\left(l-\frac{s+1}{2}\right)}\left(z_{a}^{2}\right) e^{-i \frac{s+1}{2} \varphi_{b}} e^{i \frac{s+1}{2} \varphi_{a}} \chi_{s} \chi_{s}^{+}\right\} .
\end{aligned}
$$

Taking $l \rightarrow l+\frac{s+1}{2}, I\left(\mathbf{x}_{b}, \mathbf{x}_{a}\right)$ becomes

$$
I\left(\mathbf{x}_{b}, \mathbf{x}_{a}\right)=2|G| \sum_{n=0}^{+\infty} \frac{(n) !}{\left(n+\kappa^{\prime} l\right) !} e^{-\frac{1}{2} z_{b}^{2}} z_{b}^{\kappa^{\prime} l} L_{n}^{\kappa^{\prime} l}\left(z_{b}^{2}\right) e^{-\frac{1}{2} z_{a}^{2}} z_{a}^{\kappa^{\prime} l} L_{n}^{\kappa^{\prime} l}\left(z_{a}^{2}\right) \sum_{l=-\infty}^{+\infty} \frac{e^{i l\left(\varphi_{b}-\varphi_{a}\right)}}{2 \pi} \sum_{s= \pm 1} \chi_{s} \chi_{s}^{+} .
$$

By making use of the known closure relation obeyed by the generalized Laguerre functions

$$
\sum_{n=0}^{+\infty} \frac{(n) !}{(n+\alpha) !} e^{-\frac{1}{2}\left(\rho_{a}+\rho_{b}\right)} \rho_{a}^{\frac{\alpha}{2}} \rho_{b}^{\frac{\alpha}{2}} L_{n}^{\alpha}\left(\rho_{a}\right) L_{n}^{\alpha}\left(\rho_{b}\right)=\delta\left(\rho_{b}-\rho_{a}\right)
$$

to obtain

$$
\begin{aligned}
I\left(\mathbf{x}_{b}, \mathbf{x}_{a}\right) & =2|G| \sum_{n=0}^{+\infty} \frac{(n) !}{\left(n+\kappa^{\prime} l\right) !} e^{-\frac{1}{2} z_{b}^{2}} z_{b}^{\kappa^{\prime} l} L_{n}^{\kappa^{\prime} l}\left(z_{b}^{2}\right) e^{-\frac{1}{2} z_{a}^{2}} z_{a}^{\kappa^{\prime} l} L_{n}^{\kappa^{\prime} l}\left(z_{a}^{2}\right) \sum_{l=-\infty}^{+\infty} \frac{e^{i l\left(\varphi_{b}-\varphi_{a}\right)}}{2 \pi} \sum_{s= \pm 1} \chi_{s} \chi_{s}^{+} \\
& =2|G| \delta\left(z_{b}^{2}-z_{a}^{2}\right) \delta\left(\varphi_{b}-\varphi_{a}\right) \mathbb{I}_{2 \times 2}=2|G| \delta\left(|G| r_{b}^{2}-|G| r_{a}^{2}\right) \delta\left(\varphi_{b}-\varphi_{a}\right) \mathbb{I}_{2 \times 2}
\end{aligned}
$$

Now considering the properties of the Dirac delta function

$$
\delta\left(|G| r_{b}^{2}-|G| r_{a}^{2}\right)=\frac{\delta\left(r_{b}-r_{a}\right)}{2|G| \sqrt{r_{b} r_{a}}}
$$

to finally get

$$
I\left(\mathbf{x}_{b}, \mathbf{x}_{a}\right)=\frac{\delta\left(r_{b}-r_{a}\right)}{\sqrt{r_{b} r_{a}}} \delta\left(\varphi_{b}-\varphi_{a}\right) \mathbb{I}_{2 \times 2}=\mathbb{I}_{2 \times 2} \delta\left(\mathbf{x}_{b}-\mathbf{x}_{a}\right)
$$

which proves the completeness of the Dirac oscillator eigenfunctions obtained above.

We close by noting that choosing the energy sign equal to that of the quantum number $s$, i.e. $\operatorname{sgn}\left(\mathcal{E}_{n, l, s}\right)=\operatorname{sgn}(s)$, is required by the supersymmetric nature of our spinors solution of the Dirac equation. This together with the quantification of the radial variable suggest to take the quantum number $n$ belongs to $\mathbb{N}$. However, in [25] the relation $\operatorname{sgn}\left(\mathcal{E}_{n, l, s}\right)=\operatorname{sgn}(n)$ is made by hand and therefore $n$ must be in $\mathbb{Z}$ to prove the completeness of the Dirac oscillator in 3-dimensional.

\section{Conclusion}

We have established a new symmetric expression to solve the problem of the relativistic confining fermion interacting with the constant magnetic field within the framework supersymmetric representation path integrals of Fradkin and Gitman [19]. This new symmetric form for global path integral 
represent the first attempt to find a general method to extract spinors in certain elegance. The energy spectrum is derived from the spectral decomposition of the causal Green function and have found to be in agreement with that obtained [15].

The solutions of the energy spectrum are obtained to be dependent on different physical parameters and quantum numbers. By inspecting the basic features of such solutions, we have showed there our system is hidden some interesting symmetries. Indeed, by considering three different configurations of the quantum numbers, we have noticed that the energy remained invariant. This properties could be used to systemically establish a theory based on such symmetries to deal with some physical phenomena like the quantum Hall effect [14].

Subsequently, we have focused on two important issues related to our system. The first one is the density of current where the radial part was found to be null, while the angular one was not and was expressed in terms of the generalized hypergeometric function. The second issue is the nonrelativistic limit that has been studied by using the standard method and therefore the corresponding solutions of the energy spectrum were recovered in accordance with [15].

To close, let us notice that the advantage of using the path integral techniques for the system under consideration is to extract the corresponding spinors from the spectral decomposition of the Green function in the simple and compact forms compared to those obtained in [15]. These forms make them suitable for variational calculations such that the currant density, the nonrelativistic limit and etc.

\section{Acknowledgments}

A.M and A.J acknowledge the financial support from King Faisal University. The present work was done under Project Number 140233, 'Path Integral Techniques for Interacting Dirac Particles'. A.M and A.J are very grateful to T. Sbeouelji for numerous helpful discussions and assistant.

\section{References}

[1] A.M.J. Schakel, Phys. Rev. D43 (1991) 1428; A. Neagu and A.M.J. Schakel, Phys. Rev. D48(1993) 1785.

[2] M.O. Goerbig and N. Regnault, Phys. Rev. B74 (2006 ) 161407.

[3] C. Töke, P.E. Lammert, J.K. Jain and V.H. Crespi, Phys. Rev. B74 (2006) 235417.

[4] D.V. Khveshchenko, Phys. Rev. B75 (2007) 153405.

[5] C. Toke and J.K. Jain, Phys. Rev. B75 (2007) 244540.

[6] A. Jellal, Nucl. Phys. B804 (2008) 361.

[7] J. Karwowski and G. Pestka, Theoretical Chemistry Accounts 118 (2007) 519.

[8] P.A. Cook, Lettere al Nuovo Cimento 1 (1971) 419.

[9] M. Moshinsky and A. Szczepaniak, J. Phys. A: Math. Gen. 22 (1989) L817-82. 
[10] A.K. Geim and K. S. Novoselov, Nat. Mater. 6 (2007) 183.

[11] K.S. Novoselov, A.K. Geim, S.V. Morozov, D. Jiang, Y. Zhang, S.V. Dubonos, I.V. Grigorieva and A.A. Firsov, Science 306 (2004) 666.

[12] A.H. Castro Neto, F. Guinea, N.M.R. Peres, K.S. Novoselov and A.K. Geim, Rev. Mod. Phys. 81(2009) 109.

[13] M. Grundmann, F. Heinrichsdorff, C. Ribbat, M.-H. Mao and D. Bimberg, Applied Physics:Lasers and Optics B69 (1999) 413; T. Pohjola, D. Boese, H. Schoeller, J. K nig and G. Sch n,Physica: Condensed Matter B284-288 (2000) 1762; D. Vanmaekelbergh and P. Liljeroth, Chem.Soc. Rev. 34 (2005) 299; J.L. West and N.J. Halas, Annu. Rev. Biomed. Eng. 5 (2003) 285; H.Arya, Z. Kaul, R. Wadhwa, K. Taira, T. Hirano and S.C. Kaul, Biochem. Biophys. Res. Commun.329 (2005) 1173; E.J. Gansen, M.A. Rowe, M.B. Greene, D. Rosenberg, T.E. Harvey, M.Y. Su,R.H. Had eld, S.W. Nam and R.P.Mirin, Nature Photonics 1 (2007) 585.

[14] R.E. Prange and S.M. Girvin, editors, "The Quantum Hall Effect", (Springer, New York 1990).

[15] A. Jellal, A.D. Alhaidari and H. Bahlouli, Phys. Rev A80 (2009) 012109.

[16] V.M. Villalba and A.A.R. Maggiolo, Eur. J. Phys. B22 (2001) 31.

[17] P.R. Holland, Foundations of Physics 16 (1986) 701.

[18] C. Alexandrou, R. Rosenfelder, and A.W. Schreiber, Phys. Rev. A59 (1998) 3.

[19] E.S. Fradkin and D.M. Gitman, Phys. Rev. D44 (1991) 3230; S. Haouat and L. Chetouani, Z. Naturforsch 62a (2007) 34 .

[20] N. Boudiaf, A. Merdaci and L. Chetouani, J. Phys. A: Math. Theor. 42 (2009) 015303; A. Merdaci, N. Boudiaf and L. Chetouani, Z. Naturforsch. 63a (2008) 283.

[21] D.M. Gitman and S.I. Zlatev, Phys. Rev. D55 (1997) 7701.

[22] I.S. Gradshteyn and I.M. Ryzhik, "Table of Integrals, Series, And Products" (Academic Press,New York 1980).

[23] O. Yilmaz, M. Saglam and Z. Z. Aydin, Concepts of Physics 4 (2007) 141.

[24] H. Benzair, M. Merad, T. Boudjedaa and A. Makhlouf, Z. Naturforsch 67a (2012) 77.

[25] Radosław Szmytkowski and Marek Gruchowski, J. Phys. A: Math. Gen. 34 (2001) 4991. 